\title{
TURBULENCE IN MICRO-CHANNELS
}

\author{
T. A. Kowalewski", S. Blonski \\ IPPT PAN, Polish Academy of Sciences, Warsaw, Poland
}

\begin{abstract}
Fluid mechanics in small channels, i.e. channels of micrometer size, is dominated by surface effects and often exhibits striking differences of flow characteristics when compared with macro scale. One of important microfluidic problems is flow destabilization and occurrence laminarturbulent transition. In this paper we describe our experimental and numerical attempts to understand growth of flow instabilities and development of turbulent structures in small channels. In the first configuration flow of water through $1 \mathrm{~mm}$ long and $0.4 \mathrm{~mm}$ high microchannel formed between two planes is investigated varying Reynolds number from 1000 to 6770 . Fluorescent traces are used for flow visualization and microPIV acquisition of temporary velocity fields. The microPIV data are used to evaluate turbulent flow characteristics. Our experimental study shows that destabilization of flow in such a micro-channel does not necessarily occurs when it is usually expected. Nearly laminar flow structure is present within the channel even for the highest investigated flow Reynolds number. These findings are confirmed by numerical simulation performed using finite volume code.

On the other hand it appears possible to achieve unstable flow pattern even for quite low Reynolds number flow regime by proper modification of the channel walls. In the second experimental and numerical study we demonstrate that appropriately chosen wall waviness of the micro-channel may lead to flow destabilization already at quite low flow Reynolds number $(\sim 100)$.
\end{abstract}

Keywords: microchannels, microPIV, turbulence, flow instability

\section{INTRODUCTION}

The advances of microfluidics allow for the manufacturing micro-reactors, micro-scaled cooling devices or small liquid atomisers. These devices are characterised by a large surface-to-volume ratio, hence provide possibility for larger heat fluxes or offer large catalytic surface for reactions. In most of these applications flow unsteadiness or turbulence is desirable, however not easy to achieve in small scales. The design and optimisation of microfluidic devices is usually based on our knowledge gained for macro scale hydrodynamics. However, it can be expected that small channel hydrodynamics has to be reconsidered in different contexts: relative large surface area can be responsible for delayed (or accelerated) laminar-turbulent transition [1-8]; wall roughness becomes elevated relative to small channel size and may influence laminar-turbulent transition [9]; appropriate wall corrugation can be used to activate flow instability and effectively diminish critical Reynolds number [10].

In spite of the existence of numerous experimental and theoretical investigations, a number of principal problems related to laminar-turbulent transition in micro scales are not fully resolved. There are contradictory data on transition from laminar to turbulent flow. Some studies indicate that the transition from the laminar to the turbulent flow in micro-scale passages takes place at critical Reynolds numbers, ranging from 300 to 2000. The reported values are lower than those known from the conventional theory for large-size tubes. In particular, $\mathrm{Wu}$ and Little $[1,2]$ experimentally observed that in glass and silicon microchannels having hydraulic diameters between $45.5 \mu \mathrm{m}$ and

\footnotetext{
* Corresponding author: Prof. T.A. Kowalewski

Phone: + (48)-22-8269803, Fax: + (48)-22-8269815

E-mail address: tkowale@ippt.gov.pl
} 
$83.1 \mu \mathrm{m}$, the flow was laminar for $\operatorname{Re}<1000$ and became fully turbulent for $\operatorname{Re}>3000$. Choi et al. [3], analysing microtubes with a hydraulic diameter of $53 \mu \mathrm{m}$ and $81.2 \mu \mathrm{m}$, indicated that the transition to turbulent flow occurs at $\mathrm{Re}=2000$. However, they found that this value decreases for smaller microchannels $\left(\operatorname{Re}=500\right.$ for $D_{h}=9.7 \mu \mathrm{m}$ and $\left.6.9 \mu \mathrm{m}\right)$. These and following measurements were performed by employing "classical" experimental methodology, i.e. detecting global effects like pressure drop, heat fluxes or mixing effectiveness. In small channels entrance effects and probe size may largely modify measurements, as indicated by Celata et al. [4]. Only quite recently it appeared possible to acquire detailed information on the temporary velocity flow fields within micro channel. Time-dependent measurements of the velocity field within microchannels by means of the microPIV technique have been performed to access the laminar/turbulent transition range [58]. These measurements, in principle, are able to resolve not only the integral information on the laminar/turbulent transition, they can, moreover, provide local information on both mean and fluctuating velocity contributions. Hence, information on the developing flow, on the spatial distribution of the mean flow, on the spatial distribution of turbulent fluctuations and stresses, and on the intermittent flow is accessible. Li et al. [5] measured time-dependent velocity fields in a rectangular microchannel of $320 \times 330 \mu \mathrm{m}^{2}$ cross section. They discuss profiles of the mean axial velocity, of the axial and transversal velocity fluctuations, and reported good agreement with findings known for macroscopic channels. Quite recently Wibel and Ehrhard [6] in their very careful microPIV study of velocity fluctuations confirmed that the laminar/turbulent transition in $133 \mu \mathrm{m}$ channels occurs in the range $1900 \leq \mathrm{Re} \leq 2220$, in reasonable agreement with macroscopic findings. Moreover, the profiles of the turbulent quantities are reported in good agreement with findings within macroscopic channels. All reported investigations deal with well developed flow in a long channel. However, due to high pressure drop only very short micro channels can be used in practical realization. In our micro-PIV study of high Reynolds number flow in very short micro channel $[7,8]$ delayed occurrence of turbulence was reported. This finding is subject of the present paper as well.

The occurrence of laminar/turbulent transition can be strongly modified by wall roughness, which becomes an issue for small channels [9]. On the other hand it was predicted by Szumbarski [10,11] that controlled wall waviness may lead to flow destabilization already at Reynolds number close to 100. The outcome of our numerical and experimental study of this remarkable feature is reported in the second part of the present paper.

\section{EXPERIMENTAL}

\section{Apparatus}

The main part of the experimental set-up consists of a microscope, a laser light source and a digital camera. The flow is observed using 10x (NA 0.3 / WD $17.30 \mathrm{~mm}$ ) microscope lens and area covered by the camera is $854 \mu m \times 683 \mu \mathrm{m}$. Short illumination time is achieved by using a pulsed light of Nd:YAG laser, delivering $30 \mathrm{~mJ}$ energy at $532 \mathrm{~nm}$ wavelength (New Wave Research, Inc.). The flow is illuminated and observed through the upper window of the channel. By traversing the field of observation in the horizontal and vertical direction, the position of the interrogated flow plane was selected. For the recording of images a high-resolution (1280 x 1024 pixels) 12 bit $P C O$ SensiCam camera was used. When coupled with the double pulse laser the system permits acquisition of two images at the minimum time interval of $200 \mathrm{~ns}$, exposition time of $5 \mathrm{~ns}$, and about $3.75 \mathrm{~Hz}$ repetition rate. The micro-PIV measurements were performed for pure water seeded with fluorescent tracers, polystyrene spheres of $2 \mu \mathrm{m}$ in diameter (Duke Scientific Inc.). The particle volumetric concentration was very low $(<0.0001 \% \mathrm{wt})$, hence they did not affect the flow structure. Unlike typical PIV methods, microPIV does not utilize a thin laser sheet to illuminate the seeding particles [12]. The whole investigated volume is flooded with the laser light using beam expander and the microscope objective. Two low pass filters, mounted between the objective and the camera, permit only the fluorescent red light to pass, while preventing the green laser light to be detected by the camera. The micro-PIV images present well detectable bright spots of the seeding 
particles. The accuracy of the velocity measurement depends on several experimental factors (quality of the images, seeding concentration, particle displacement), as well as on the vector evaluation procedure. Using in house developed software and by evaluating uniform, predefined flow of water through the micro-channel, the error of velocity measurement was estimated to be below $5 \%$.

In most cases the flow was driven by the micro pump (Cole-Palmer Inst.) permitting flow rate variation from $\mathrm{Q}_{\mathrm{V}}=0.02 \mathrm{~cm}^{3} / \mathrm{s}$ to $70 \mathrm{~cm}^{3} / \mathrm{s}$. For the highest measured flow rates $\left(200 \mathrm{~cm}^{3} / \mathrm{s}\right)$ pressurized supplying system was used [7].

\section{Short channel turbulence}

The flow of pure water was studied using in a flat model of an emulsifier, described already in details $[7,8]$. It consists of a small channel formed between two glass plates and a triangular processing element creating the rapid flow contractions (Fig. 1). Dimension of the two gaps created between glass plates and processing element (triangular obstacle) are $0.4 \mathrm{~mm}$ in height, $1 \mathrm{~mm}$ in length and $15 \mathrm{~mm}$ in width. The flat geometry and two glass windows permit application of optical methods for measuring flow velocity fields (microPIV method) within the gap $\left(\mathrm{P}_{1}, \mathrm{P}_{1-2}, \mathrm{P}_{2}\right)$ as well as nearly $4 \mathrm{~mm}$ upstream $\left(\mathrm{P}_{0}\right)$ and $3 \mathrm{~mm}, 5 \mathrm{~mm}$, and $8 \mathrm{~mm}$ downstream $\left(\mathrm{P}_{3}, \mathrm{P}_{4}, \mathrm{P}_{5}\right)$. The flow rate used varied from 16 to $204 \mathrm{~cm}^{3} / \mathrm{s}$, and the corresponding Reynolds number based on the microchannel height varied from 1000 - 6770. For the highest flow rate the both symmetrical microchannels (upper and lower gap) were supplied, whereas in other cases only interrogated upper gap was open for the flow.
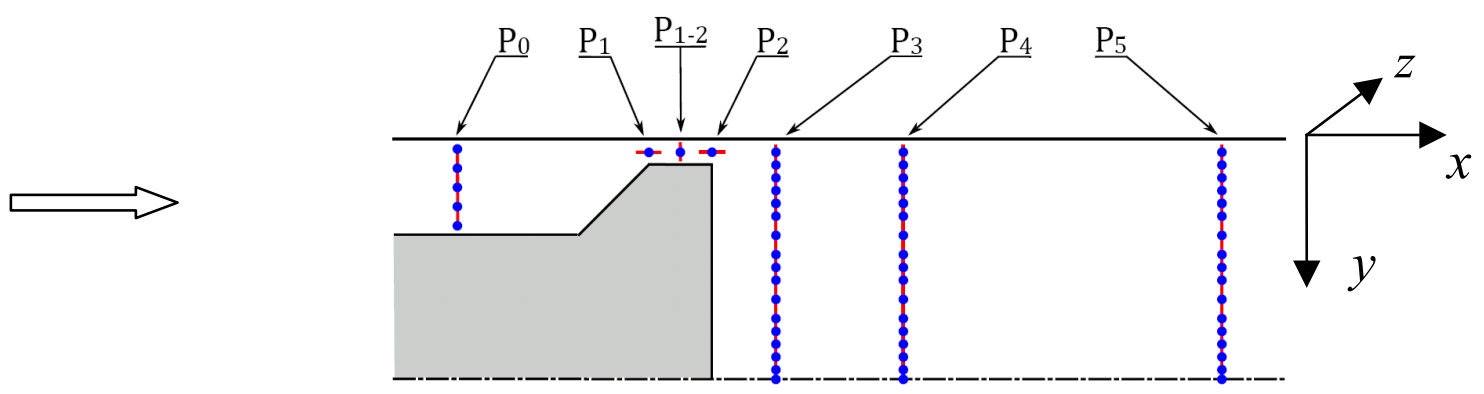

Figure 1. Schematic drawing of the channel and locations $\mathrm{P}_{0}-\mathrm{P}_{5}$ interrogated by the PIV method. The flow is driven from left to right. Distances are measured from the gap exit $(x=0)$ and the top wall $(y=0)$. At location $\mathrm{P}_{0}(x=-4 \mathrm{~mm})$ PIV covers 5 planes between top and bottom wall; at $\mathrm{P}_{1}$ and $\mathrm{P}_{2}$ PIV interrogation planes are in the mid-height of the gap $(y=-0.2 \mathrm{~mm})$, covering partly gap inlet and outlet, respectively; at $\mathrm{P}_{1-2}$ measurements are located in the middle of the length of the microchannel $(x=-0.5 \mathrm{~mm})$ and cover three vertical positions $y=-0.1 \mathrm{~mm}, y=-0.2 \mathrm{~mm}$ and $y$ $=-0.3 \mathrm{~mm} . \mathrm{P}_{3}-\mathrm{P}_{5}$ indicate location of PIV interrogation planes used to measure velocity distribution from the top wall to the flow axis $(y=0:-3.75 \mathrm{~mm})$. They are located at distances $x=$ $1 \mathrm{~mm}\left(\mathrm{P}_{3}\right), x=3 \mathrm{~mm}\left(\mathrm{P}_{4}\right)$, and $x=8 \mathrm{~mm}\left(\mathrm{P}_{5}\right)$ from the gap exit.

\section{Corrugated wall}

A simple model of the wavy channel formed between two plates has been machined in polycarbonate using micro-machining technique. The average channel height is $793 \mu \mathrm{m}$, its width is $33.6 \mathrm{~mm}$, and the length $75 \mathrm{~mm}$. Surface of the bottom wall is modulated by 20 rows (comp. Fig. 2). They create spanwise periodic structure with channel depth varying from $0.4 \mathrm{~mm}$ to $1 \mathrm{~mm}$. The upper wall of the channel is flat to permit optical measurements. An average flow velocity in the corrugated channel varied from about $0.76 \mathrm{~mm} / \mathrm{s}$ to $2.6 \mathrm{~m} / \mathrm{s}$, which corresponds to Reynolds number based on the average channel height $R e=0.6$ and $R e=2100$, respectively. The channel length is probably too short to allow for fully developed flow instabilities to occur spontaneously. However, it is assumed that initial spanwise flow disturbances generated by the strongly divergent flow inlet may become amplified by the wall waviness, if the theoretical prediction $[10,11]$ is correct. The 
microPIV velocity measurements and the flow visualisation of single fluorescent traces are done. These experiments aim identification of the critical Reynolds number. The microPIV measurements provide quantitative data about development of flow field disturbances. Long time exposure of single fluorescent traces are used to indicate possible spatial fluctuations of observed streaklines.
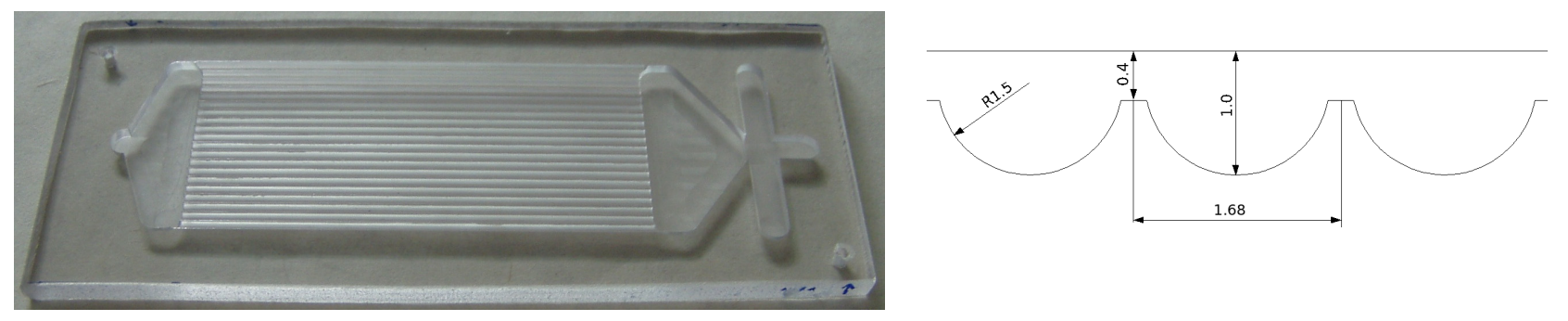

Figure 2. Left: experimental channel with 20 spanwise wall corrugations. The top wall is flat for better optical access; right: cross section of the channel with dimensions of the corrugations (in $\mathrm{mm})$.

\section{NUMERICAL}

Finite-volume package Fluent 6.3 (ANSYS Inc.) is used to generate the computational grid and to perform stability analysis using unmodified three-dimensional set of Navier-Stokes equations (DNS). The DNS model allows obtaining accurate, unsteady solution of unmodified Navier-Stokes equations by resolving the whole range of spatial and temporal scales of the turbulence. In our case the main aim of the simulation is to identify presence of instability modes and the critical Reynolds number for their amplification. All the spatial scales of the turbulence must be resolved in the computational mesh. Hence, very fine mesh and small time steps are used (typical $10^{-7} \mathrm{~s}$ ). The direct numerical simulation (DNS) performed with the classical finite volume code implemented in Fluent is time consuming and vulnerable. Nevertheless, it appeared that for both investigated geometries it was possible to obtain reasonable solutions reproducing typical for the turbulence flow characteristics.

Short microchannel. The numerical domain used in the simulations of flow in the short microchannel describes full 3D geometry of the whole device, including $97.5 \mathrm{~mm}$ long inlet and $78.5 \mathrm{~mm}$ long outlet. Preliminary estimation of the Kolmogorov scales shows that smallest flow structures should be about $1 \mu \mathrm{m}$ in size with time scale below $1 \mu \mathrm{s}$. According to this estimation structural hexahedron mesh with boundary layer was generated in the gap and in the vicinity of the processing element. The tetrahedron mesh was used in the remaining parts of the computational domain. It was found that sufficient accuracy of the numerical model was obtained for the gap mesh of 50x60x50 nodes (smallest distance between gap mesh nodes was about $3 \mu \mathrm{m}$ ), fine enough to resolve expected Kolmogorov microscale. The total number of computational cells for the whole domain was over $1.7 \mathrm{mln}$. Detailed parameters used for modelling flow in the short microchannel are those collected in [8]. Simulations performed covered four flow rates, corresponding to the microchannel Reynolds number 1000, 1800, 3300 and 6770.

Corrugated wall. Numerical simulation of instabilities growth in the channel must assume long enough (theoretically infinite) channel length. Hence, computational domain used for simulating flow in the corrugated channel assumed periodic boundary conditions for the inlet and outlet. For the spanwise direction two sets of performed computations included either periodic boundary conditions or two side walls limiting computational domain [11]. For the first case (fully periodic boundary conditions) total number of elements was about $0.4 \mathrm{mln}$. Inclusion of side walls increased this number from $0.7 \mathrm{mln}$ for five corrugation periods to $23.4 \mathrm{mln}$ elements for full 20 periods of wall corrugation. The last configuration reproduces the experimental geometry. However, due to 
prohibitive computational time most of the numerical solutions are obtained for fully periodic computational domain or domain with five periods of corrugation limited by two side walls.

\section{RESULTS}

\section{Short microchannel}

In order to quantify the turbulence in the channel, the ensemble-averaged velocity fields are calculated at each of seven selected location before, within, and behind the microchannel (Fig. 1). Measurements were repeated up to hundred times at the same position to obtain sufficient statistics [8]. With 5 flow rates, 70 areas interrogated by microPIV measurements, and in average 50-100 measurements taken for each position, the total number of evaluated flow fields exceeds 50,000 .

Velocity measurements performed at two positions $\mathrm{P}_{1}$ and $\mathrm{P}_{2}$ within the gap indicate that the flow through the gap is practically laminar, even for the highest applied flow rate no large temporal flow field fluctuations could be found. The streamwise velocity of the flow rapidly increases in the vicinity of the entrance to the gap, but turbulent fluctuations remain low despite high flow Reynolds number (6770). Velocity measurements at the gap exit $\left(\mathrm{P}_{2}\right)$ show development of the first instabilities (Fig. 3a). Behind the gap streamwise flow velocity decreases and strong recirculation zone with the reversal of flow can be found in the region behind the processing element $[7,8]$. The turbulent fluctuations of the velocity field and break-up of the flow symmetry, observed in this region, indicate that probably transition from laminar to turbulent flow regime occurs there.
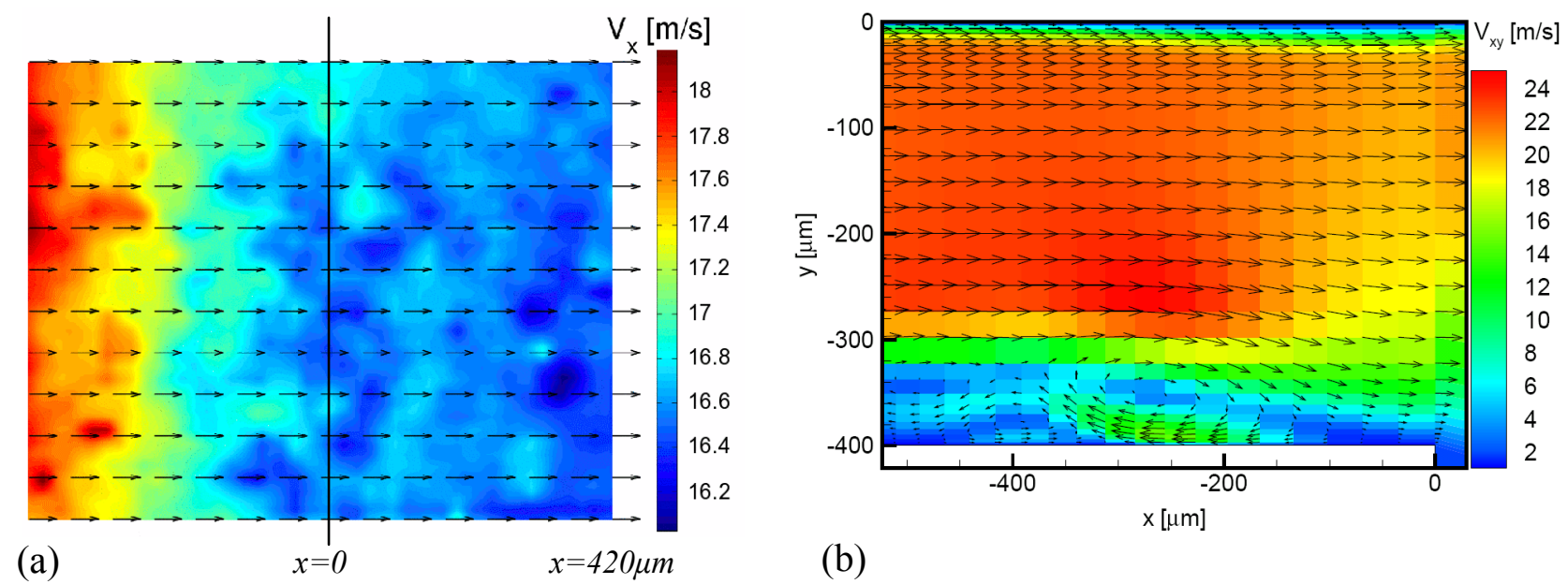

(b)

Figure 3. (a) - flow structure measured by micro-PIV at the $x$-z horizontal plane located in the midheight $(y=-0.2 \mathrm{~mm})$ of the outlet region of microchannel (half of the field covers region in the microchannel and half behind it). (b) - temporary flow structure at the vertical symmetry plane of outlet region of the microchannel obtained form DNS simulation. Contours coloured by the velocity magnitude.

Numerical modelling confirms main details of the velocity flow field measured by the micro-PIV method. For the highest flow rate a small vortex in the bottom part of microchannel outlet region (Fig. 3b) could be found. It indicates place where flow destabilization is initiated, propagating downstream to the outer region. Both experimental data (Fig. 4a) and DNS simulations (Fig. 4b) indicate that intensity of turbulence (turbulence energy $\mathrm{tke}_{\mathrm{xz}}$ ) remains relatively low before and in the microchannel, rapidly increasing downstream from the gap. However, the turbulent kinetic energy values estimated form the numerical model and from the PIV measurement are only in a qualitative agreement (Fig.4b). Visible quantitative differences are believed to be mainly due to limited accuracy of the micro-PIV measurements and missing data for the third velocity component (comp. [7,8]). 
The numerical data are used to re-evaluate Kolmogorov microscales within the microchannel. It appears that even for the highest Reynolds number Kolmogorov length and time scales amount within the gap about $1 \mathrm{~mm}$ and $1 \mathrm{~s}$, respectively. It confirms experimental findings that in spite of relatively high Reynolds number the velocity fluctuations are very small and flow within the microchannel seems to be laminar.

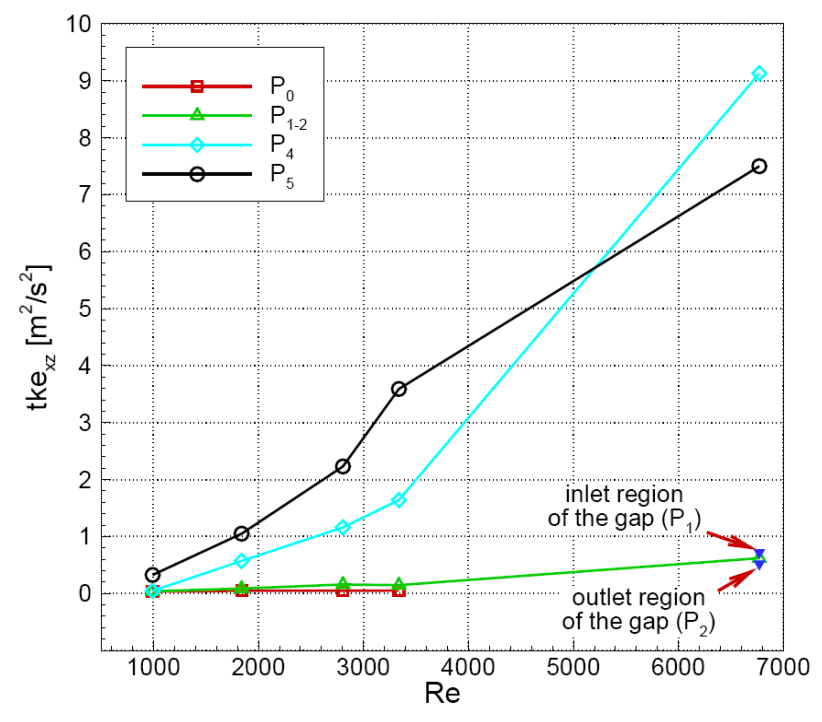

(a)

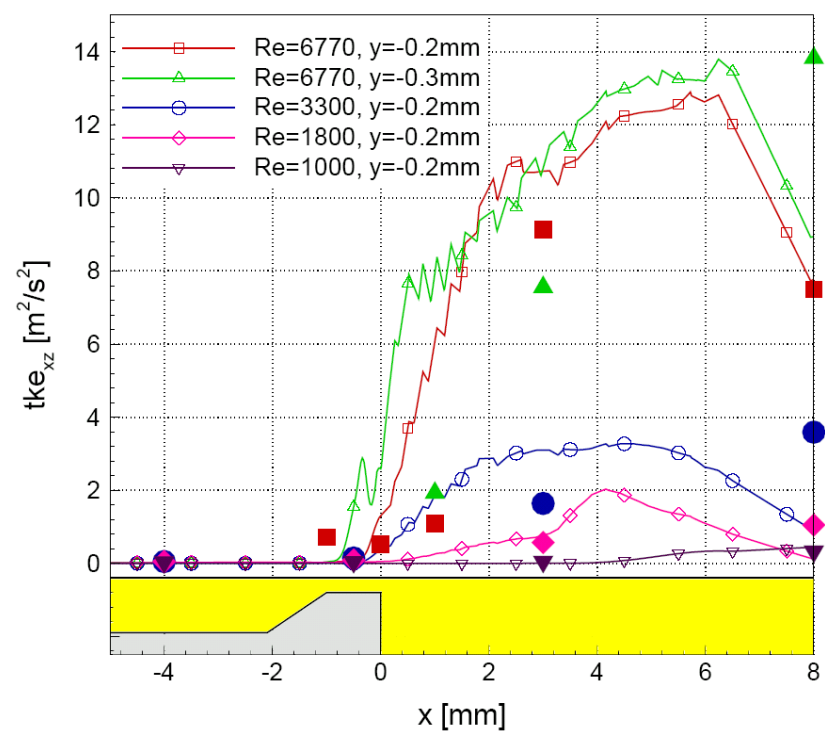

(b)

Figure 4. (a) - turbulent kinetic energy tke $\mathrm{x}_{\mathrm{xz}}$ as a function of Reynolds number evaluated from the PIV data $0.2 \mathrm{~mm}$ below the top wall $(y=-0.2 \mathrm{~mm})$ at 4 different locations along the experimental model $\left(\mathrm{P}_{0}-\mathrm{P}_{5}\right)$; (b) - variation of turbulent kinetic energy tke $\mathrm{x}_{\mathrm{xz}}$ evaluated from DNS results along the computational domain, given for two vertical positions: $y=-0.2 \mathrm{~mm}$ (red line) and $y=-0.3 \mathrm{~mm}$ (green line) below the top wall. Filled symbols indicate corresponding experimental data.

\section{Corrugated wall}

The transversal flow disturbances are generated across the whole channel width, interacting in a complex way with all 20 corrugations (Fig. 2). Flow pattern in the corrugated channel was visualized using long time exposure $(120 \mathrm{~ms})$ of single fluorescent traces. These experiments allow for fast identification of the critical Reynolds number. At low flow rates the particle tracks exhibit straight lines. Increasing flow rate the wavy character of particle tracks reveals emerging transversal flow disturbances. Our preliminary experimental study [11] shows that transversal velocity fluctuations can be visualized by particle tracking at least for the flow Reynolds number $R e=120$ (Fig. 5). The wavy motion of the tracer implies presence of the transversal velocity component.
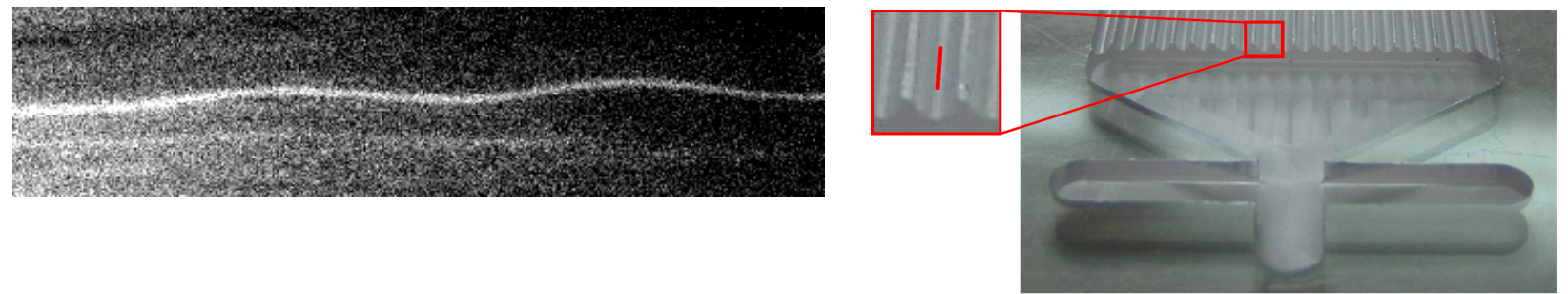

Figure 5: Left: trace of the fluorescent particle recorded in the corrugated channel (Fig. 2). The image width corresponds to $0.3 \mathrm{~mm}$, illumination time is $0.12 \mathrm{~s}$, flow Reynolds number $R e=120$. Right: position in the channel, where particle trace was recorded. 
Micro-PIV measurements performed within the channel were used to obtain the flow disturbances. Proper understanding of the full structure of the analysed flow needs recombination of several sets of single flow fields obtained at different locations across the channel, and at its different depths. The typical disturbed velocity field structure obtained by means of micro-PIV is given in Fig. 6a. The mean flow is subtracted to reveal transversal velocity components. Due to the small interrogated flow area $(0.85 \mathrm{~mm} \times 0.68 \mathrm{~mm})$ only flow in the vicinity of a single corrugation can be shown. Nevertheless, one may find that local spanwise flow disturbances are present, indicating emerging flow instabilities.

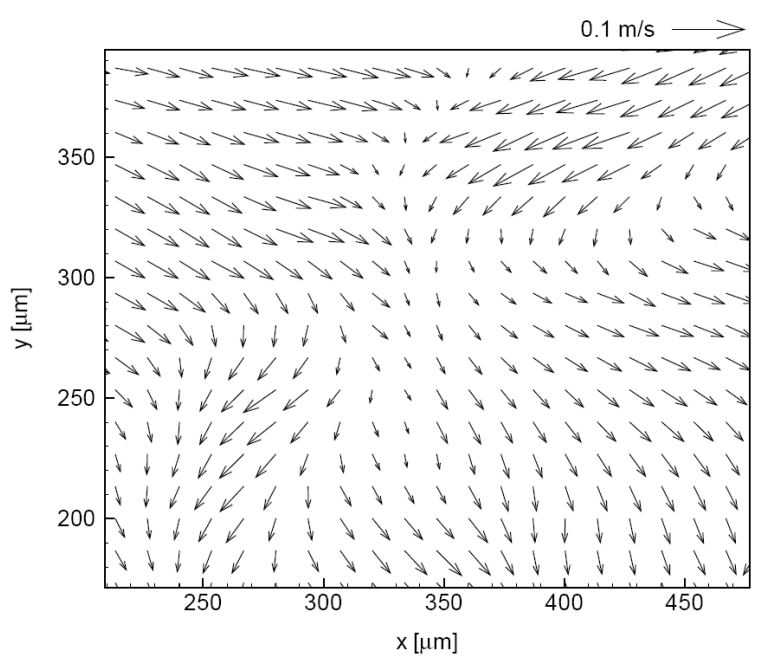

(a)

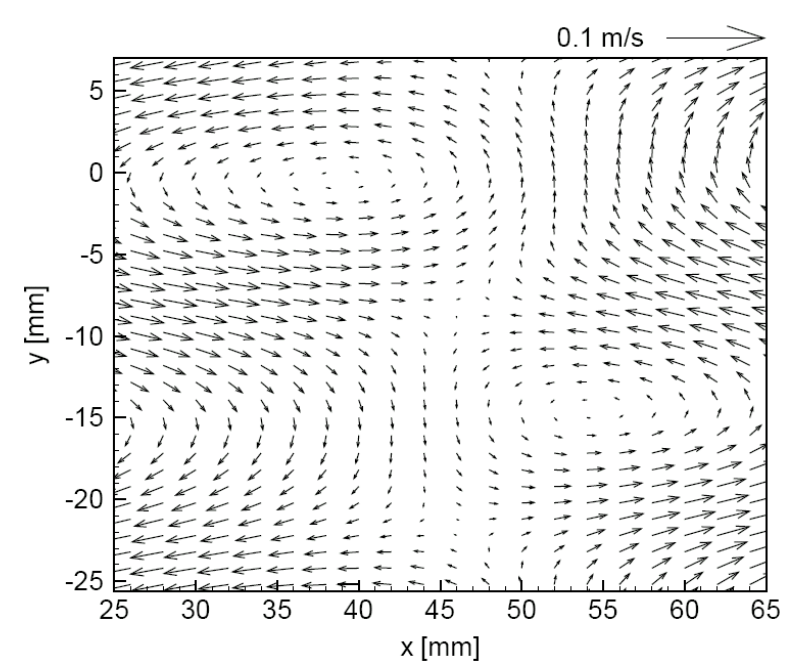

(b)

Figure 6. (a) Velocity field fluctuations measured in the corrugated channel $0.2 \mathrm{~mm}$ below the upper wall, Reynolds number $R e=200$; (b) - flow fluctuation predicted by the numerical simulations for the symmetry plane, flow Reynolds number $R e=100$.

Numerical simulations performed for the corrugated channel confirmed generation of transversal instability modes for flow Reynolds number as low as 100 . It was found that for the flow with Reynolds number $R e=100$ small initial flow disturbances are quickly amplified and after about $200 \mathrm{~s}$ fully developed unsteady flow is observed [11]. The flow velocity field extracted from the numerical solution for the channel central cross-section (Fig. 6b) well reproduces predicted by the linear stability analysis space periodic counter-rotating vortices [10] travelling in the streamwise direction. It seems evident that the introduced wall waviness generates spanwise instabilities propagating along the channel and disturbing the flow structure. The new unstable flow pattern, which emerges form the unstable mode have complex three-dimensional structure promoting mixing properties of the channel flow. It is interesting to note that numerical model indicates that presence of the side walls (confined channel) does not significantly change this picture.

\section{CONCLUSIONS}

Experimental and numerical investigations demonstrated that flow pattern within the short, nearly twodimensional microchannel remains laminar even for Reynolds number well above 6000 . Obviously small channel dimensions are responsible for damping the development of classical cascade of turbulent vortices. This fact may have an important impact on design of emulsifiers [8], or microchannel heat exchangers. On the other hand it is shown that proper modification of the microchannel walls may lead to flow destabilization already at Reynolds numbers about 100 . This effect predicted by the theoretical study [10] is confirmed both numerically and experimentally. 


\section{NOMENCLATURE}

tke $\quad=\left\langle V_{x z}^{\prime 2}\right\rangle+\left\langle V_{z}^{\prime 2}\right\rangle$, Turbulent Kinetic Energy for $x-z$ horizontal plane $\left[\mathrm{m}^{2} \mathrm{~s}^{-2}\right]$.

Re Reynolds number based on the channel height.

$Q_{V} \quad$ volumetric flow rate $\left[\mathrm{m}^{3} \mathrm{~s}^{-1}\right]$.

$V_{x, y, z}, V_{x, y, z}^{\prime}$ components of the velocity and of the fluctuating part of the velocity, respectively.

$x, y, z \quad$ coordinates along the channel, across the channel, and perpendicular to channel crosssection in Fig. 1; $x-z$ PIV interrogated planes.

\section{ACKNOWLEDGMENTS}

This investigation was supported by the Polish Ministry of Science and Education under project Enhancement of the mixing process in micro-flows, grant No.: N501008733.

\section{REFERENCES}

1. Wu P., Little W. A., Measurement of Friction Factors for the Flow of Gases in Very Fine Channels used for Microminiature Joule-Thompson Refrigerators, Cryogenics, vol. 23, pp. 273277, 1983.

2. Wu P., Little W.A., Measurement of Heat Transfer Characteristics of Gas Flow in Fine Channel Heat Exchangers Used for Microminiature Refrigerators, Cryogenics, vol. 24, pp. 415-420, 1984.

3. Choi S.B., Barron R. F., Warrington R. O., Liquid Flow and Heat Transfer in Microtubes, Micromechanical Sensors, Actuators and Systems, ASME DSC, vol. 32, pp. 123-128, 1991.

4. Celata G.P., Cumo M., McPhail S., Zummo G., Characterization of Fluid Dynamic Behavior And Channel Wall Effects in Microtubes, Int. J. Heat Fluid Flow, vol. 27, pp. 135-143, 2006.

5. Li H., Ewoldt R., Olsen M.G, Turbulent and Transitional Velocity Measurements in a Rectangular Microchannel Using Microscopic Particle Image Velocimetry, Exp. Therm. Fluid Scie., vol. 29, pp.435-446, 2005.

6. Wibel W., Ehrhard P., Experiments on the Laminar/turbulent Transition of Liquid Flows in Rectangular Microchannels. Heat Transfer Eng., vol. 30 pp. 70-77, 2009.

7. Kowalewski T.A., Błoński S., Korczyk P., Turbulent Flow in a Microchannel, Proc. of ASME ICNMM2006, CD-ROM paper 96090, pp. 1-10, University of Limerick, Ireland, 2006.

8. Blonski S., Korczyk, P.M., Kowalewski T.A., Analysis of Turbulence in a Micro-channel Emulsifier, Int. J. Therm. Scie., 46, pp. 1126-1141, 2007.

9. Kandlikar S.G., Roughness Effects at Microscale - Reassessing Nikuradse's Experiments on Liquid Flow in Rough Tubes. Bull. Pol. Ac.: Tech., vol. 53, pp. 343-349, 2005.

10. Szumbarski J., Instability of Viscous Incompressible Flow in a Channel with Transversely Corrugated Walls, Journal of Theoretical and Applied Mechanics 45(3), pp. 659-683, 2007

11. Kowalewski T.A, Szumbarski J., Blonski S., Low-Reynolds-Number Instability of the Laminar Flow between Wavy Walls, Proc. of ASME ICNMM2008, CD-ROM proceedings ISBN 0-79183826-9, paper 62070, pp.1-8, TU Darmstadt, Germany, 2008.

12. Santiago J.G., Wereley S.T., Meinhart C.D., Beebe D.J., Adrian R.J., A Micro Particle Image Velocimetry System, Exp. Fluids, vol. 25, pp. 316-319, 1998. 